\title{
EFFECTIVE MATCHMAKING AND $k$-CHROMATIC GRAPHS
}

\author{
ALFRED B. MANASTER ${ }^{1}$ AND JOSEPH G. ROSENSTEIN ${ }^{2}$
}

ABSTRACT. In an earlier paper we showed that there is a recursive society, in which each person knows exactly two other people, whose marriage problem is solvable but not recursively solvable. We generalize this result, using a different construction, to the case where each person knows exactly $k$ other people. From this we deduce that for each $k \geqq 2$ there is a recursive $2(k-1)$-regular graph, whose chromatic number is $k$ but which is not recursively $k$ chromatic.

1. Graphs, societies, and algorithms. Following Berge [1] a set $S$ of unordered pairs of distinct elements of a set $P$ determines a graph $\Gamma=$ $(P, S)$. The elements of $P$ are called points or vertices of $\Gamma$; the elements of $S$ are called $\operatorname{arcs}$ of $\Gamma$. It is not assumed that $P$ or $S$ is finite. Points $x$ and $y$ are said to be adjacent if $\{x, y\}$ is an arc. $\Gamma$ is $k$-chromatic if the points of $\Gamma$ can be painted with $k$ colors in such a way that no two adjacent points are of the same color. The chromatic number of $\Gamma$ is the smallest number $k$ such that $\Gamma$ is $k$-chromatic. $\Gamma$ is $k$-regular if every point of $\Gamma$ is adjacent to exactly $k$ points. $\Gamma$ is called simple (or bipartite) if there exist disjoint sets $B$ and $G$ such that $P=B \cup G$ and if wherever $\{x, y\} \in S$ then $x \in B$ and $y \in G$ or $y \in B$ and $x \in G$. Two distinct arcs are said to be adjacent if they have a point in common. A matching of a simple graph $(B, G, S)$ is a set $W$ of arcs no two of which are adjacent. Let $W$ be a matching, $B_{I V}=\{b \in B \mid$ for some $g,\{b, g\} \in W\}$, and $G_{W}=\{g \in G \mid$ for some $b,\{b, g\} \in W\} ; W$ is then said to be a matching of $B_{W}$ onto $G_{W}$ or a matching of $B_{W}$ into $G$.

We now recall the more colorful, anthropomorphic terminology of Halmos and Vaughan [3]. Let $\Sigma=(B, G, S)$ be a simple graph. We call

Presented in part to the Society, January 24, 1971 under the title Effective matchmaking (Recursion theoretic aspects of a theorem of Philip Hall); received by the editors August 23, 1972.

AMS (MOS) subject classifications (1970). Primary 02F50, 05C15, 02E10; Secondary $05 \mathrm{~A} 05$.

Key words and phrases. Bipartite graph, $k$-regular graph, chromatic number, matching, marriage problem, algorithm, recursive function.

${ }^{1}$ Partially supported by NSF Grant GP-23771.

2 Partially supported by NSF Grant GP-20134.

(c) American Mathematical Society 1973 
$\Sigma$ a society, $B$ the set of boys, and $G$ the set of girls. If $b$ and $g$ are adjacent in the graph $\Sigma$ we say that $b$ and $g$ are acquainted in the society $\Sigma$. We call the society $\Sigma$ a $k$-society if as a graph it is $k$-regular, so that in a $k$ society each person knows exactly $k$ people of the opposite sex. The society $\Sigma$ is said to have a solvable marriage problem if there is a matching of $B$ into $G$, for we can think of the matching as providing, in a monogamous way, a mate for each boy from among the girls he knows. Similarly, $\Sigma$ is said to have a symmetric solution to its marriage problem if there is a matching of $B$ onto $G$

We also associate with the society $\Sigma$ another graph $\Gamma_{\Sigma}$ as follows. The points of $\Gamma_{\Sigma}$ are the arcs of $\Sigma$ and the arcs of $\Gamma_{\Sigma}$ are the unordered pairs of adjacent arcs of $\Sigma$. If $\Sigma$ is a $k$-society then $\Gamma_{\Sigma}$ is a 2(k-1)-regular graph.

We use three combinatorial lemmas which we state here without proof.

LeMmA 1. If $\Sigma$ is a $k$-society, then there is a symmetric solution to its marriage problem.

Lemma 2. If $\Sigma$ is a $k$-society, the chromatic number of $\Gamma_{\Sigma}$ is $k$.

Lemma 3. Let $\Sigma$ be a $k$-society. The set of points of $\Gamma_{\Sigma}$ possessing a common color in a k-coloring of $\Gamma_{\Sigma}$ is a matching of $B$ onto $G$ in $\Sigma$. Thus such a set of points is a solution to the symmetric marriage problem of $\Sigma$.

In the finite case, Lemmas 1 and 2 are just restatements of results due to König and P. Hall appearing in Berge [1, pp. 92-95]. To prove these lemmas in the infinite case, one can, for example, use $O$. Ore's extension of the Schroeder-Bernstein theorem (Theorem 1.3.4 in Mirsky [4]). Lemma 3 is easily verified directly.

Following Rogers [5] a function which is computable by an algorithm or an effective procedure is called a partial recursive function. The domain of a partial recursive function is assumed to be a subset of $N^{m}$ for a fixed $m$ ( $N$ is the set of natural numbers) and its range is assumed to be a subset of $N$. If its domain happens to be all of $N^{m}$ the partial recursive function $\theta$ is called a (general) recursive function. If $x$ is in the domain of $\theta$ we say that $\theta(x)$ is defined; otherwise we say that $\theta(x)$ is undefined. A set is said to be recursive if its characteristic function is a recursive function.

The collection of all finite sets of instructions, or algorithms, formulated in a fixed language can be recursively (i.e. effectively) enumerated. Assuming this to be done, $\phi_{e}$ denotes the partial recursive function defined by the $e$ th finite set of instructions. Given an argument $x$ and a number $e$ of a set of instructions, it is not possible to determine effectively whether or not $\phi_{e}(x)$ is defined. However, it is possible, for each $n$, to determine effectively-in $e, x$, and $n$-whether or not $\phi_{e}(x)$ is defined in $n$ steps, by simply carrying out $n$ steps of the $e$ th algorithm applied to $x$ and 
observing the outcome. " $\phi_{e}^{n}(x)$ is defined" will mean that $\phi_{e}(x)$ is defined in $n$ steps; in that case the value of $\phi_{e}^{n}(x)$ will be $\phi_{e}(x)$. Furthermore if $\phi_{e}(x)$ is defined there must be an $n$ such that $\phi_{e}^{n}(x)$ is defined-and for all $n^{\prime} \geqq n, \phi_{e}^{n^{\prime}}(x)$ is defined and equals $\phi_{e}(x)$. The formal statements and verifications of these remarks can be found, for example, in Rogers [5, Theorems 1-IV and 1-IX].

In what follows a society will also satisfy the conditions (i) each person knows only finitely many other people (i.e. $\Sigma$ is locally finite) and (ii) everyone knows someone. If all but (ii) are satisfied by $\Sigma$, then $\Sigma$ will be called a partial society. The connected components of a partial society $\Sigma$ are called the communities of $\Sigma$.

We say that the society $\Sigma$ is recursive if $B$ is the set of even natural numbers, $G$ is the set of odd natural numbers, and $S$ is a recursive set of ordered pairs. We will use $B(n)$ for $2 n$ and $G(n)$ for $2 n+1$ and say that $B(n)$ is the $n$th boy and that $G(n)$ is the $n$th girl. A recursive society is said to be recursively (symmetrically) solvable if there is a 1-1 (onto) recursive function $f$ such that, for each $n, B(n)$ knows $G(f(n))$.

We say that the graph $\Gamma=(P, S)$ is recursive if $P=N$ and $S$ is a recursive set of ordered pairs. $\Gamma$ is said to be recursively $k$-chromatic if there is a recursive function $f$ of one variable whose range is a subset of $\{0,1, \cdots, k-1\}$ such that if $x$ is adjacent to $y$ then $f(x) \neq f(y)$.

Let $\Sigma$ be a recursive society and let $j$ be a 1-1 function which maps $S$ recursively onto $N$. Define $j\left(\Gamma_{\Sigma}\right)$ to be the graph whose points are $N$ and whose arcs are the pairs $\left\{j(b, g), j\left(b^{\prime}, g^{\prime}\right)\right\}$ such that $\left\{(b, g),\left(b^{\prime}, g^{\prime}\right)\right\}$ is an arc of $\Gamma_{\Sigma}$. Observe that if $\Sigma$ is a recursive society, $j\left(\Gamma_{\Sigma}\right)$ is a recursive graph. Since $j\left(\Gamma_{\Sigma}\right)$ is isomorphic to $\Gamma_{\Sigma}$, we know that if $\Sigma$ is a $k$-society, $j\left(\Gamma_{\Sigma}\right)$ is a $2(k-1)$-regular graph, and that, by Lemma $2, j\left(\Gamma_{\Sigma}\right)$ has chromatic number $k$. Lemma 3 shows that if $j\left(\Gamma_{\Sigma}\right)$ is recursively $k$-chromatic, then $\Sigma$ is recursively solvable. These observations show that the following corollary is a consequence of the existence of a recursive $k$-society which is not recursively solvable. This will be proved in $\$ 2$.

COROLLARY. There is a recursive $2(k-1)$-regular graph whose chromatic number is $k$, but which is not recursively $k$-chromatic.

\section{Recursive $k$-societies without recursive solutions.}

THEOREM. For each $k \geqq 2$ there is a recursive $k$-society which is symmetrically solvable but is not recursively solvable.

Proof. In the proof we construct a recursive society $\Sigma$ by stages; at stage $n$, for each $n>0$, a partial society $\Sigma_{n}=\left(B, G, S_{n}\right)$, with $S_{n}$ finite, is effectively defined so that, for each $n, S_{n} \subseteq S_{n+1}$ and so that $\Sigma=$ $\left(B, G, \cup_{n>0} S_{n}\right)$ has the desired properties. Instead of saying "put $(x, y)$ 
into $S_{n}$ " we will say "introduce $x$ to $y$ " or "introduce $y$ to $x$ " at stage $n$. A person is called a "stranger" at a given point in the construction if he has not yet been introduced to anyone. At the beginning of each stage $n$ of the construction there are numbers $a$ and $b$ (with $a \geqq n$ and $b \geqq n$ ) such that the first $a$ boys and $b$ girls are not strangers at that point, but the remaining boys and girls are; we will reserve the numbers $a$ and $b$ for this purpose, so that $B(a)$ and $G(b)$ always are the first male and female strangers at the beginning of the appropriate stage of the construction.

For each $n$, each introduction made during stage $n$ involves at least one person who was a stranger at the beginning of stage $n$. This feature, together with the effectiveness of the construction of $S_{n}$, implies that $\Sigma$ is recursive. To see this we show how to decide whether or not $B(p)$ knows $G(q)$. Let $n>p$ and $n>q$. Since the first male and female strangers at stage $n$ are $B(a)$ and $G(b)$ where $a \geqq n$ and $b \geqq n$ it follows that $B(p)$ and $G(q)$ have acquaintances in $\Sigma_{n}$. Hence $B(p)$ knows $G(q)$ in $\Sigma$ if and only if he already knows her in $\Sigma_{n}$. But whether or not he knows her in $\Sigma_{n}$ can be effectively determined by effectively reconstructing $S_{n}$.

The community of the partial society $\Sigma_{n-1}$ to which $B(i)$ belongs at the beginning of stage $n$ will be denoted $C_{n}(i) . C_{n}(i)$ is called stable if $C_{m}(i)=$ $C_{n}(i)$ for all $m \geqq n$. The remarks in the preceding paragraph show that if $C_{n}(i)$ is stable, then no member of $C_{n}(i)$ will ever meet someone new. In particular, if $C_{n}(i)$ is stable and $B(p)$ and $G(q)$ are in $C_{n}(i)$ but cannot marry in $C_{n}(i)$ (i.e. there is no solution to the marriage problem of $C_{n}(i)$ in which $B(p)$ marries $G(q))$, then $B(p)$ cannot marry $G(q)$ in $\Sigma$.

We now define simultaneously the recursive society $\Sigma$ and $k 1-1$ recursive functions $r_{0}, r_{1}, \cdots, r_{k-1}$ (with pairwise disjoint ranges); at the end of stage $n, r_{t}(i)$ will be defined for all $i<n$ and $t<k$.

Intuitively, the construction will guarantee that if $\phi_{e}\left(r_{t}(e)\right)$ is defined for all $t<k$ than no solution to the marriage problem of $\Sigma$ simultaneously marries each $B\left(r_{t}(e)\right)$ to the corresponding $G\left(\phi_{e}\left(r_{t}(e)\right)\right)$, so that $\phi_{e}$ cannot be a solution to the marriage problem of $\Sigma$. Since every recursive function is $\phi_{e}$ for some $e$, this implies that the marriage problem of $\Sigma$ has no recursive solution.

We assume as part of the induction hypothesis that at stage $n$ for each $i<n$ either all $B\left(r_{t}(i)\right)$ are in the same community or they are in $k$ different communities. In the former case, the community is a stable one in which each person knows exactly $k$ others. In the latter case there is a $p$ such that for each $t<k$ the community $C_{n}\left(B\left(r_{t}(i)\right)\right)$ contains exactly $1+(k-1) k+$ $(k-1)^{3} k+\cdots+(k-1)^{2 p+1} k$ boys and $k+(k-1)^{2} k+\cdots+(k-1)^{2 p} k$ girls, and can be put into 1-1 correspondence with the nodes of the graph $g_{p}$ below in such a way that boys correspond to nodes marked $\delta$, girls correspond to nodes marked $q, B\left(r_{t}(i)\right)$ corresponds to the bottom node, 

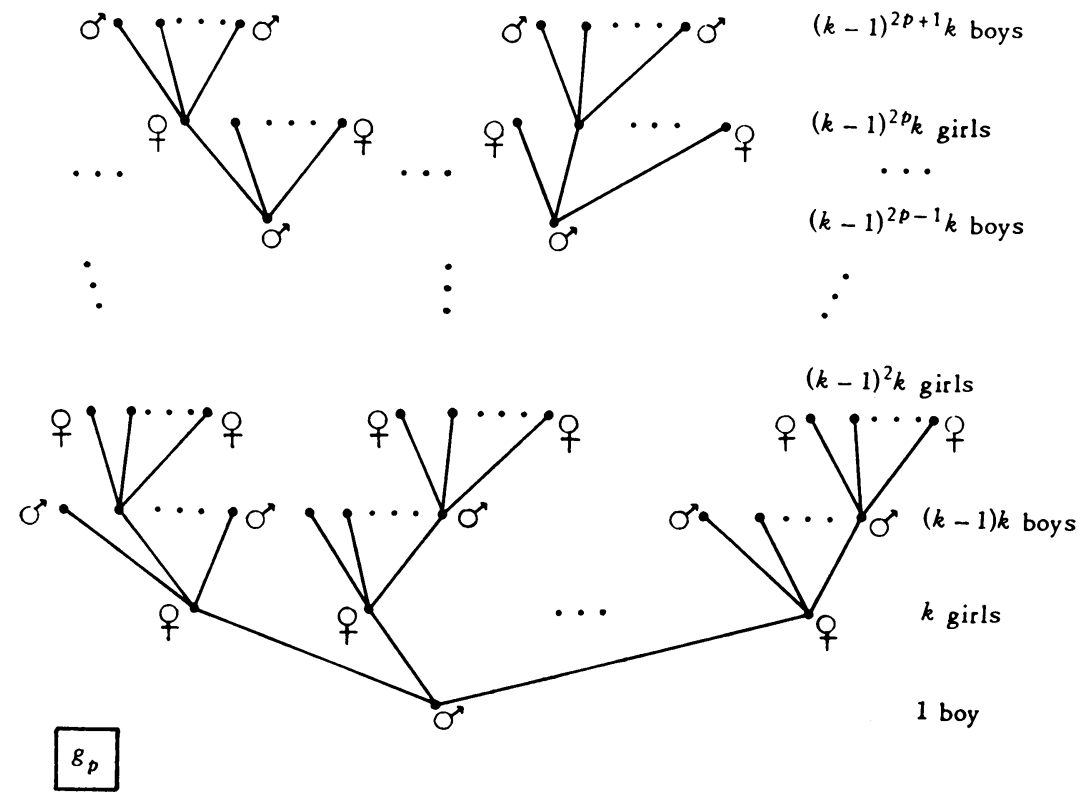

FIGURE 1

and two nodes are adjacent if and only if the people mapped to these nodes know each other. If this is the case we shall say that $C_{n}\left(B\left(r_{t}(i)\right)\right)$ has form $g_{p}$. We assume that the definition of the $j$ th row of $g_{p}$, for $0 \leqq j \leqq 2 p+2$, and of the $i$ th position (from the left) on the $j$ th row of $g_{p}$, for $0 \leqq i<(k-1)^{j-1} k$ where $j \geqq 1$, need not be made explicit. It is also clear what we mean when we say that (under a particular correspondence) a certain person of the community $C$ (which has form $g_{p}$ ) is in the $i$ th position of the $j$ th row of $C$. Note that in a community of form $g_{p}$ each person except those on the top, i.e. $(2 p+2)$ th, row know exactly $k$ other people.

Stage $n>0$. Define $r_{t}(n-1)=a+t$ for each $t<k$ (the first $k$ unused boys) and establish for each $B\left(r_{t}(n-1)\right)$ a community containing $k$ additional new girls, and $k(k-1)$ additional new boys, so that it has the form $g_{0}$.

Let $n=2^{e} q$ where $q$ is odd, say $q=2 m+1$. If all $B\left(r_{t}(e)\right)$ are already in the same community proceed to stage $n+1$. If they are still in different communities, and if either some $\phi_{e}^{n}\left(r_{t}(e)\right)$ is undefined or all are defined but some $B\left(r_{t}(e)\right)$ does not know $G\left(\phi_{e}^{n}\left(r_{t}(e)\right)\right)$, then, since each of the $k$ communities is of the form $g_{p}$ where $p=m-1$, we introduce each of the $(k-1)^{2 p+1} k$ boys in the top row of each community to $k-1$ new girls and each of these $(k-1) \cdot(k-1)^{2 p+1} k$ girls to $(k-1)$ new boys, so that 
the resulting $k$ communities are all of the form $g_{p+1}$. Finally, we consider the case where the $B\left(r_{t}(e)\right)$ are in different communities, where all $\phi_{e}^{n}\left(r_{t}(e)\right)$ are defined, and where $B\left(r_{t}(e)\right)$ knows $G\left(\phi_{e}^{n}\left(r_{t}(e)\right)\right)$ for each $t<k$. We assume that each $C_{n}\left(B\left(r_{t}(e)\right)\right)$ has the form $g_{p}$ and that the correspondence between $C_{n}\left(B\left(r_{t}(e)\right)\right)$ and the nodes of $g_{p}$ places $G\left(\phi_{e}^{n}\left(r_{t}(e)\right)\right)$ in the leftmost, i.e. 0th, position in the first row of $C_{n}\left(B\left(r_{t}(e)\right)\right)$, for each $t<k$. [At most a relabelling is necessary to guarantee this.] Let $B_{i}^{t}$ be the boy in the $i$ th position of the top row of $C_{n}\left(B\left(r_{t}(e)\right)\right)$ for each $i<T=(k-1)^{2 p+1} k$ and each $t<k$. Let $G_{0}, G_{1}, \cdots, G_{T(k-1)-1}$ denote the first $T(k-1)$ female strangers. Introduce $B_{i}^{t}$ to each of $G_{i}, G_{T+i}, G_{2 T+i}, \cdots, G_{(k-2) T+i}$ for each $t<k$ and each $i<T$.

This completes the construction of $\Sigma$. Before we proceed to prove that it satisfies the desired properties we shall consider the following situation which contains within it the essence of the argument.

Suppose then that $k=3$ and that $e$ is such that at stage $n=2^{e}$ we have that $\phi_{e}^{n}\left(r_{t}(e)\right)$ is defined for each $t<3$ and that $B\left(r_{t}(e)\right)$ knows $G\left(\phi_{e}^{n}\left(r_{t}(e)\right)\right)$ for each $t<3$. At this stage each $C_{n}\left(B\left(r_{t}(e)\right)\right)$ has the form $g_{0}$. After rearrangement these communities take the form

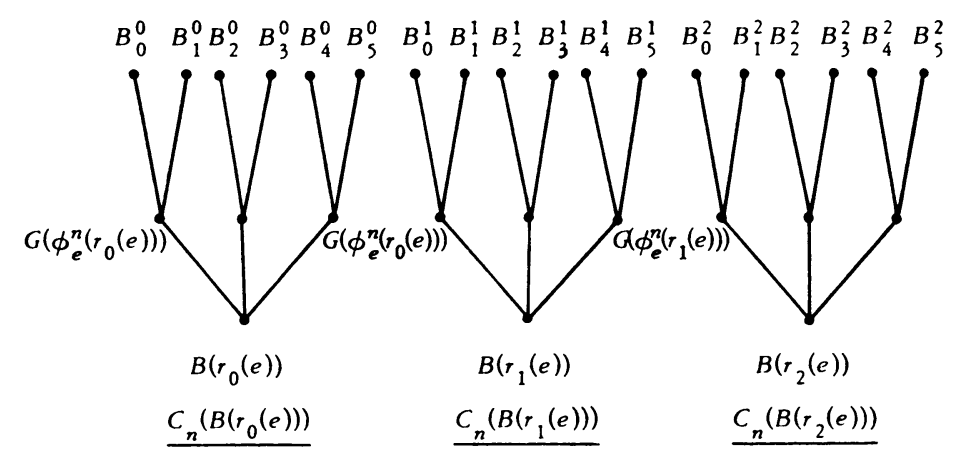

Thus the final case of the construction is the relevant one. After it is applied, we obtain the community $C_{n+1}\left(B\left(r_{0}(e)\right)\right)$ which assumes the form below. It is now evident that in no solution to the marriage problem of $C_{n+1}\left(B\left(r_{0}(e)\right)\right)$ can $B\left(r_{t}(e)\right)$ marry $G\left(\phi_{e}^{n}\left(r_{t}(e)\right)\right)$ for each $t<3$. For, of $B_{0}^{0}$, $B_{0}^{1}, B_{0}^{2}$ exactly two marry $G_{0}$ and $G_{6}$; the remaining one $B_{0}^{t^{*}}$ must marry $G\left(\phi_{e}^{n}\left(r_{t^{*}}(e)\right)\right)$ so that $B\left(r_{t^{*}}(e)\right)$ cannot marry her. Similarily, of $B_{1}^{0}, B_{1}^{1}, B_{1}^{2}$ exactly two marry $G_{1}$ and $G_{7}$; the remaining one $B_{1}^{\text {t\# }}$ must marry $G\left(\phi_{e}^{n}\left(r_{t} \#(e)\right)\right)$ so that $B\left(r_{t} \#(e)\right)$ cannot marry her. Hence, in fact, only (and exactly) one of $B\left(r_{t}(e)\right)$ marries $G\left(\phi_{e}\left(r_{t}(e)\right)\right.$ ).

We return now to the general case. It is clear from the construction that $\Sigma$ is a recursive society (note that each introduction involves a stranger) and that each community of $\Sigma$ is either finite, in which case each person 


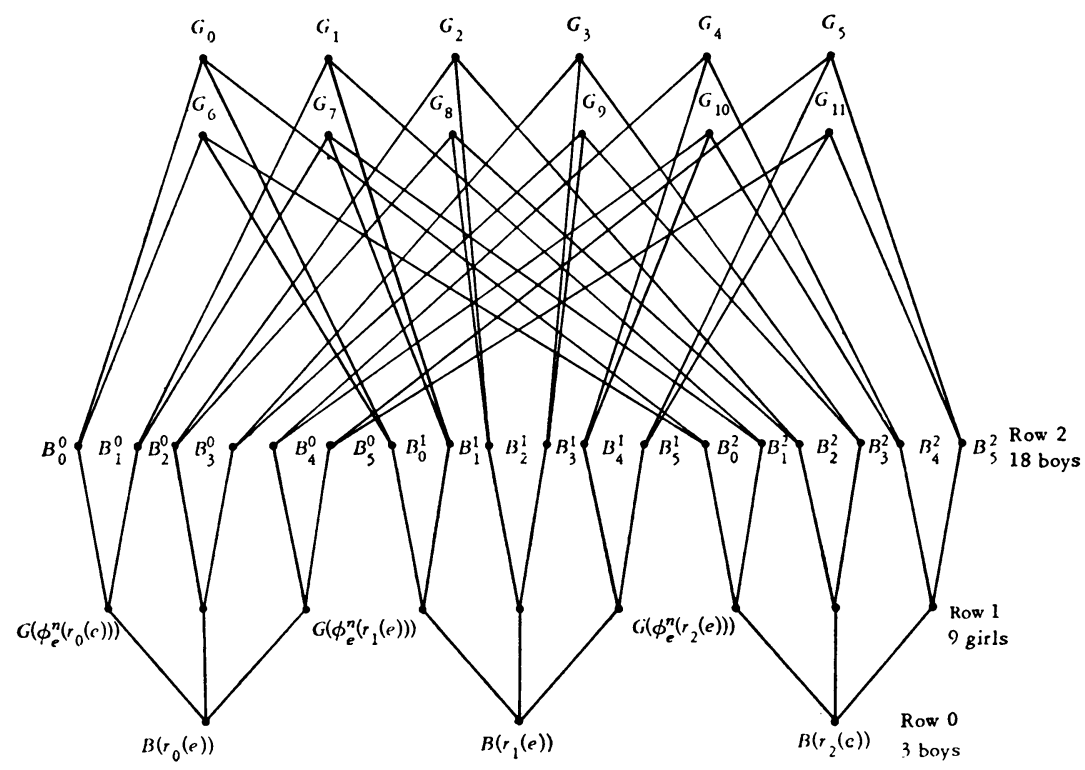

in it knows exactly $k$ others, or has form $g$ where $g$ is the direct limit of the graphs $g_{p}$; so again each person in it knows exactly $k$ other people. That $\Sigma$ is symmetrically solvable follows from Lemma 1 .

Thus we need only show that $\Sigma$ is not recursively solvable-i.e. that no $\phi_{e}$ is a solution to the marriage problem of $\Sigma$. It suffices, of course, to show that if $\phi_{e}\left(r_{t}(e)\right)$ is defined for each $t<k$ and $B\left(r_{t}(e)\right)$ knows $G\left(\phi_{e}\left(r_{t}(e)\right)\right)$ for each $t<k$ then no solution to the marriage problem of $C\left(B\left(r_{0}(e)\right)\right)$ marries each $B\left(r_{t}(e)\right)$ to the corresponding $G\left(\phi_{e}\left(r_{t}(e)\right)\right)$. Note of course that under these hypotheses at some stage $n$ we combine the $C_{n}\left(B\left(r_{t}(e)\right)\right)$ into one community which is stable at stage $n$. We may assume that at stage $n$ each $C_{n}\left(B\left(r_{t}(e)\right)\right)$ has the form $g_{p}$ (for some $p$ ) and that $G\left(\phi_{e}\left(r_{t}(e)\right)\right)$ is in the leftmost position in the first row of $C_{n}\left(B\left(r_{t}(e)\right)\right)$.

We shall show, by induction on $j<2 p+3$ that for each $i$ if $A_{t}$ is the person in the $i$ th position of the $(2 p+3-j)$ th row of $C_{n}\left(B\left(r_{t}(e)\right)\right)$ for $t<k$ then in any solution to the marriage problem of $C_{n}\left(B\left(r_{t}(e)\right)\right)$ exactly one of $\left\{A_{t} \mid t<k\right\}$ marries a person on the row below-i.e. on the $(2 p+2-j)$ th row. Thus taking $j=2 p+2$ and $i=0$ we conclude that exactly one of $G\left(\phi_{e}\left(r_{t}(e)\right)\right)$ marries $B\left(r_{t}(e)\right)$, hence certainly not every $G\left(\phi_{e}\left(r_{t}(e)\right)\right)$ marries $B\left(r_{t}(e)\right)$.

For $j=0$ we must consider, for each fixed $i$, the boys $B_{i}^{0}, B_{i}^{1}, \cdots, B_{i}^{k-1}$ on the top row. Now at stage $n$ each of these $k$ boys was introduced to the $k-1$ girls $G_{i}, G_{T+i}, G_{2 T+1}, \cdots, G_{(k-2) T+i}$. In any fixed solution to the marriage problem some $B_{i}^{t}$ must marry a girl other than these; but the 
only other girl he knows is on the row below him. Also, since we added $\left[(k-1)^{2 p+1} k\right] \cdot(k-1)$ girls at stage $n$, the total number of girls in $C_{n+1}\left(B\left(r_{0}(e)\right)\right)$ is $\left[k+(k-1)^{2} k+\cdots+(k-1)^{2 p} k\right] k+(k-1)^{2 p+2} k$ which equals the total number of boys

$$
\left[1+(k-1) k+(k-1)^{3} k+\cdots+(k-1)^{2 p+1} k\right] k
$$

in $C_{n+1}\left(B\left(r_{0}(e)\right)\right)$, so that any solution to the marriage problem of $C_{n+1}\left(B\left(r_{0}(e)\right)\right)$ is symmetric. Hence each $G_{p T+i}$ must marry one of $B_{i}^{t}$. Hence exactly one $B_{i}^{t}$ marries a girl on the row below him.

Now assume that the claim is proven for $j<2 p+3$ and suppose that $j+1<2 p+3$. Then each $A_{t}$ in the $i$ th position of the $(2 p+2-j)$ th row knows exactly $k-1$ people on the row above the $(2 p+3-j)$ th row, and these people are in the $(i(k-1))$ th, $(i(k-1)+1)$ th, $\cdots,(i(k-1)+(k-2))$ th positions on the $(2 p+3-j)$ th row. Now exactly one of the people in the $(i(k-1)+s)$ th position marries a person below him, for each $s<k-1$. Thus exactly $k-1$ of $A_{0}, A_{1}, \cdots, A_{k-1}$ marry people in the row above them. Hence exactly one of them marries a person in the row below. This completes the induction and the proof.

\section{REFERENCES}

1. Claude Berge, The theory of graphs and its applications, Methuen, London; Wiley, New York, 1962. MR 24 \#A2381.

2. A. B. Manaster and J. G. Rosenstein, Effective matchmaking (Recursion theoretic aspects of a theorem of Phillip Hall), Proc. London Math. Soc. (3) 25 (1972), 615-654.

3. P. Halmos and H. Vaughan, The marriage problem, Amer. J. Math. 72 (1950), 214-215. MR 11, 423.

4. L. Mirsky, Transversal theory, Academic Press, New York, 1971.

5. Hartley Rogers, Jr., Theory of recursive functions and effective computability, McGraw-Hill, New York, 1967. MR 37 \#61.

Department of Mathematics, University of California at San Diego, la Jolla, CALIFORNIA 92037

Department of Mathematics, Rutgers University, The State University of New Jersey, New Brunswick, New Jersey 08903 